\title{
LARGE DISCRETE SETS IN STEIN MANIFOLDS
}

\author{
JÖRG WINKELMANN
}

\begin{abstract}
We construct infinite discrete subsets of Stein manifolds with remarkable properties. These generalize results of Rosay and Rudin on discrete subsets of $\mathbb{C}^{n}$.
\end{abstract}

\section{INTRODUCTION}

Rosay and Rudin constructed examples of discrete subsets in $\mathbb{C}^{n}$ with rather remarkable properties (see [8]):

1. There exists a discrete subset $D \subset \mathbb{C}^{n}$ such that the identity map is the only automorphism of $\mathbb{C}^{n}$ stabilizing $D$.

2. There exists a discrete subset $D \subset \mathbb{C}^{n}$ such that for every nondegenerate holomorphic map $F: \mathbb{C}^{n} \rightarrow \mathbb{C}^{n}$ the image $F\left(\mathbb{C}^{n}\right)$ has non-empty intersection with $D$.

3. There are uncountably many inequivalent discrete subsets of $\mathbb{C}^{n}$ (where two discrete subsets $D_{1}, D_{2}$ are called equivalent if there exists an automorphism $\phi$ of $\mathbb{C}^{n}$ such that $\left.\phi\left(D_{1}\right)=D_{2}\right)$.

Recently, Kaliman, using similar methods, showed the following (

4. There exists a discrete subset $D \subset \mathbb{C}^{n}$ such that $\mathbb{C}^{n} \backslash D$ is measurehyperbolic.

(Measure hyperbolicity is a notion introduced by Eisenman in [3], see also [5].)

The goal of this article is to generalize these constructions to complex manifolds other than $\mathbb{C}^{n}$. On a first glance it might appear that $\mathbb{C}^{n}$ is the most complicated case and that therefore similar statements for arbitrary manifolds should be easy corollaries of the statements for $\mathbb{C}^{n}$. However, this is not the case. Indeed, there are simple counterexamples to properties 1,2 und 4 .

Proposition 1 (see ex. 1, ex. 2, ex. 3). 1. Let $V$ be a Stein manifold, $X=\mathbb{P}_{n} \backslash\{[1: 0: 0]\}$ with $n=\operatorname{dim} V>0$ and $D \subset X$ a discrete subset.

Then there exists a non-degenerate holomorphic mapping $F$ : $V \rightarrow X \backslash D$ and $X \backslash D$ is not measure hyperbolic.

1991 Mathematics Subject Classification. AMS Subject Classification: 32E10, $32 \mathrm{H} 02$. 
2. Let $D$ be a discrete subset of $X=\mathbb{C} \times \mathbb{P}_{1}$.

Then there exists a non-trivial automorphism $\phi$ of $X$ with $\left.\phi\right|_{D}=$ $i d_{D}$.

Thus it is necessary to impose some conditions in order to generalize these statements to complex manifolds other than $\mathbb{C}^{n}$.

Analyzing the methods of Rosay and Rudin, we came to the conclusion that the crucial property of $\mathbb{C}^{n}$ is the existence of an exhaustion by taut relatively compact domains, namely balls. Given such an exhaustion it is possible to replace arguments using the affine-linear structure of $\mathbb{C}^{n}$ by arguments based on the tautness of these relatively compact domains, i.e. normal family arguments. Manifolds admitting such an exhaustion are called "weakly Stein" in this article. Here is the precise definition:

Definition. A complex manifold $X$ is called weakly Stein if there exists a plurisubharmonic exhaustion function $\rho: X \rightarrow \mathbb{R}_{0}^{+}$such that

1. For every $c>0$ the set $B_{c}=\{x \in X: \rho(x)<c\}$ is taut.

2. $S_{c}=\{x \in X: \rho(x)=c\}$ has empty interior for all $c$.

In the sequel, we will simply say that $(Y, \rho)$ is weakly Stein in order to indicate that $Y$ is a weakly Stein manifold and $\rho$ a plurisubharmonic exhaustion function on $Y$ with the properties required in the definition. Every Stein manifold is weakly Stein (see §2).

We prove the generalization of the results of Rosay, Rudin and Kaliman.

Theorem 1. Let $X$ be a weakly Stein manifold, and let $V$ be an irreducible affine variety with $\operatorname{dim} V=\operatorname{dim} X>0$.

Then the following assertions hold:

1. There exists a discrete subset $D \subset X$ such that $\phi(D) \neq D$ for every automorphism $\phi$ of $X$ except the identity.

2. There exists a discrete subset $F \subset X$ such that $F(V) \cap D \neq\{\}$ for every non-degenerate holomorphic map $F: V \rightarrow X$.

3. There exist uncountably many inequivalent discrete subsets of $X$.

4. There exists a discrete subset $D \subset X$ such that $X \backslash D$ is measure hyperbolic.

The first assertion is theorem 2, the second theorem 4, the third theorem 3 and the last one is theorem 5 .

\section{WEAKLY StEIN MANIFOLDS}

Let $X$ be a Stein manifold. Then there exists an embedding $i: X \hookrightarrow$ $\mathbb{C}^{n}$ and $\rho(x) \stackrel{\text { def }}{=}\|i(x)\|^{2}$ defines a plurisubharmonic exhaustion function 
on $X$. For every $c>0$ the open subset $B_{c}=\{x \in X: \rho(x)<c\}$ is biholomorphic to a closed submanifold of a ball in $\mathbb{C}^{n}$ and therefore taut. It is obvious that $S_{c}=\{x \in X: \rho(x)=c\}$ has empty interior. Thus a Stein manifold is necessarily weakly Stein.

More generally we have the following:

Lemma 1. Let $X$ be a complex manifold. Then the following four conditions are equivalent:

1. The complex manifold $X$ is both weakly Stein and holomorphically convex

2. $X$ is holomorphically convex and every compact complex analytic subspace of $X$ is hyperbolic (in the sense of Kobayashi).

3. There exists a Stein space $Z$ and a proper surjective holomorphic map $\pi: X \rightarrow Z$ such that all fibers are hyperbolic.

4. For every infinite discrete subset $D \subset X$ and for every nonconstant holomorphic map $g: \mathbb{C} \rightarrow X$ there exists a holomorphic function $f$ on $X$ such that $f$ is unbounded on $D$ resp. $g(\mathbb{C})$.

Proof. First recall that a complex manifold $X$ is holomorphically convex if and only if for every discrete subset $D$ there exists a holomorphic function $f$ such that $\left.f\right|_{D}$ is unbounded and that this property is equivalent to the existence of a proper, surjective, connected holomorphic map from $X$ onto a Stein space $Z$. Using this, it is easy to check that each of the four properties implies that $X$ is holomorphically convex and that there is a proper, surjective, connected holomorphic map $\pi: X \rightarrow Z$ onto a Stein space $Z$.

Now let $W$ be a compact complex analytic subspace of a weakly Stein manifold $(X, \rho)$. Then $W$ is contained in some $B_{c}=\{x \in X: \rho(x)<$ $c$ \}, because $W$ is compact. Thus $W$ is a closed subspace of a taut manifold and therefore taut and in particular hyperbolic. Hence compact complex analytic subspaces of weakly Stein manifolds are hyperbolic and therefore (1) implies (2).

Since $\pi: X \rightarrow Z$ is proper, its fibers are compact. Thus (2) implies (3).

Assume (3). Since $Z$ is Stein, it admits a strictly plurisubharmonic exhaustion function $\tau$. For each $c>0$ the open set $U_{c}=\{z \in Z$ : $\tau(z)<c\}$ is complete hyperbolic. Since $\pi$ is proper, the relative version of Brody's theorem (see [6]) implies that $B_{c}=\{x \in X: \pi(\tau(x))<c\}$ is complete hyperbolic and therefore taut for every $c \in \mathbb{R}$. Hence (3) implies that $(X, \rho \circ \pi)$ is weakly Stein. Furthermore, hyperbolicity of the $\pi$-fibers implies that $\pi \circ g: \mathbb{C} \rightarrow Z$ can not be constant for any non-constant holomorphic map $g: \mathbb{C} \rightarrow X$. Since $Z$ is Stein, it follows that for every non-constant holomorphic map $g: \mathbb{C} \rightarrow X$ there exists 
a non-constant holomorphic function $f_{0}$ on $Z$ such that $f_{0} \circ \pi \circ g$ is non-constant on $\mathbb{C}$. Thus (3) implies (4).

Finally observe that condition (4) implies that there is no nonconstant holomorphic map from $\mathbb{C}$ into a fiber of $\pi$. By Brody's theorem ([2]) it follows that the fibers of $\pi$ are hyperbolic. Hence (4) implies (3).

There are also weakly Stein manifolds which are not holomorphically convex. Namely, let $M$ be a projective hyperbolic manifold and $L$ a flat line bundle on $M$ such that none of the $L^{n}$ with $n \in \mathbb{Z} \backslash\{0\}$ is holomorphically trivial. Let $\|\cdot\|$ be a compatible hermitian metric. Then $\|\cdot\|$ is a plurisubharmonic exhaustion function having all the required properties. Thus $L$ is weakly Stein. On the other hand there are no non-constant holomorphic functions on $L$. Hence the non-compact complex manifold $L$ is not holomorphically convex.

\section{Preparations}

We begin by translating resp. slightly generalizing two technical results of [8]. For this we utilize the following elementary lemma.

Lemma 2. Let $(Y, \rho)$ be a weakly Stein manifold with the corresponding exhaustion function, $X$ a connected complex manifold of the same dimension and $F: X \rightarrow Y$ be a non-degenerate (i.e. having maximal rank at some point) holomorphic map. Assume that $F(X) \subset\{y \in Y$ : $\rho(y) \leq c\}$.

Then $F(X) \subset\{y \in Y: \rho(y)<c\}$.

Proof. By the maximum principle for plurisubharmonic functions it follows that either the conclusion of the lemma holds or $F(Y) \subset\{y \in$ $Y: \rho(y)=c\}=S_{c}$. However, the latter possibility is excluded by the assumptions that $F$ is non-degenerate and that $S_{c}$ has empty interior.

The following is a translation of lemma 4.3. of [8].

Lemma 3. Let $U_{2}$ be a complex manifold, $U_{1} \subset U_{2}$ a relatively compact connected open subset and $C \subset U_{1}$ a compact subset. Furthermore let $(Y, \rho)$ be weakly Stein, $0<C_{1}<C_{2}, V_{i}=\left\{y \in Y: \rho(y)<C_{i}\right\}$ and $K \subset V_{1}$ be a compact subset. Moreover fix hermitian metrics on $U_{2}$ and $V_{2}$ and a real constant $c>0$.

Let $\Gamma$ denote the set of all holomorphic mappings $F$ from $U_{2}$ to $V_{2}$ such that $F(C) \cap K \neq \emptyset$ and $\|D F\|_{C} \geq c$ (where $\|D F\|_{C}$ is the supremum over all $\|D F\|_{x}$ for $x \in C$, calculated with respect to the choosen Hermitian metrics). Fix also a natural number $m \in \mathbb{N}$. 
Then there exists a finite set $E=E\left(C, U_{1}, U_{2} ; K, V_{1}, V_{2} ; c, m\right) \subset \partial V_{1}$ with the following property:

If $F \in \Gamma$ then either $F\left(U_{1}\right) \subset V_{1}$ or $F\left(U_{2}\right) \cap E$ contains at least $m$ points.

Proof. For the case $m=1$ the proof goes exactly as in [8] (using lemma 2 instead of the maximum principle). Observe that the method of construction allows one to require that $E$ is contained in a fixed dense subset of $\partial V$. Hence, after having constructed one such set $E$ we may construct another one, disjoint to the first one. Continuing in this way, we may construct $m$ such sets and the union of all these $m$ sets is the desired set $E$.

We now slightly generalize lemma 4.4. of [B].

Lemma 4. Let $U_{2}$ be a complex manifold, $U_{1} \subset U_{2}$ a relatively compact connected open subset, $C \subset U_{1}$ a compact subset, $c>0,(Y, \rho)$ be a weakly Stein manifold, $r_{n}$ an strictly increasing sequence of positive numbers with $\lim r_{n}=+\infty, V_{n}=\left\{y \in Y: \rho(y)<r_{n}\right\}, K \subset V_{1}$ a compact subset and assume that both $U_{2}$ and $Y$ are equipped with hermitian metrics. Let $\Gamma$ denote the set of all holomorphic mappings $F: U_{2} \rightarrow Y$ such that $F(C) \cap K \neq \emptyset$ and $\|D F\|_{C} \geq c$. Let $m \in \mathbb{N}$.

Then there exists a discrete subset $E^{\prime}=E^{\prime}\left(C, U_{1}, U_{2} ; K, V_{1}, Y ; c, m\right)$ of $Y \backslash V_{1}$ such that for every $F \in \Gamma$ either $F\left(U_{1}\right) \subset V_{1}$ or $F\left(U_{2}\right) \cap E^{\prime}$ contains at least $m$ points.

Proof. Choose an increasing sequence of connected open subsets $W_{i} \subset$ $\left.U_{2}(i \in \mathbb{N})\right)$ such that

1. Every $W_{k}$ is relatively compact in $W_{k+1}$

2. $W_{1}=U_{1}$

3. The union of all $W_{k}$ is relatively compact in $U_{2}$.

Let $E^{\prime}=\cup_{k \geq 1} E_{k}$ with

$$
E_{k}=E^{\prime}\left(C, W_{k}, W_{k+1} ; K, V_{k}, V_{k+1} ; c, m\right)
$$

Evidently $E^{\prime}$ is discrete and $E^{\prime} \cap V_{1}=\emptyset$. Since $\cup_{k} W_{k}$ is relatively compact in $U_{2}$, for every $F \in \Gamma$ there exists a number $n \in \mathbb{N}$ such that $F\left(W_{k}\right) \subset V_{n}$ for all $k$. In particular $F\left(W_{n}\right) \subset V_{n}$. Let us assume that \# $\left(F\left(U_{2}\right) \cap E^{\prime}\right)<m$. Clearly \# $\left(F\left(U_{2}\right) \cap E^{\prime}\right)<m$ implies $\#\left(F\left(W_{n}\right) \cap E_{n-1}\right)<m$. Hence lemma 3 yields $F\left(W_{n-1}\right) \subset F\left(V_{n-1}\right)$. Going down by induction, we end up with the conclusion $F\left(U_{1}\right)=$ $F\left(W_{1}\right) \subset F\left(V_{1}\right)$ for all $F \in \Gamma$ with $\#\left(F\left(U_{2}\right) \cap E^{\prime}\right)<m$. 
Proposition 2. Let $X$ be a hermitian complex manifold with an increasing sequence of open connected subsets $U_{k}$ such that each $U_{k}$ is relatively compact in $U_{k+1}$. Furthermore let $(Y, \rho)$ be a weakly Stein manifold equipped with a hermitian metric, $r_{n}$ an strictly increasing sequence of positive numbers with $\lim r_{n}=+\infty, V_{n}=\left\{y \in Y: \rho(y)<r_{n}\right\}$ and $\operatorname{dim}_{\mathbb{C}}(X)=\operatorname{dim}_{\mathbb{C}}(Y)$.

Then there exists a discrete subset $D \subset Y$ and a mapping $\alpha: \mathbb{N} \times$ $\mathbb{N} \times \mathbb{R}^{+} \rightarrow \mathbb{N}$ with the following property:

Let $F: X \rightarrow Y$ be a non-degenerate holomorphic map such that there is a number $M \in \mathbb{N}$ such that $\#\left(F\left(U_{n+1}\right) \cap D\right) \leq M+\#\left(V_{n} \cap D\right)$ for all $n \in \mathbb{N}$.

Then $F\left(U_{n}\right) \subset V_{n}$ for all $n \geq \alpha(M, q, c)$ where $q=\min \{k \geq 2$ : $\left.F\left(\overline{U_{k}}\right) \cap \overline{V_{k}} \neq \emptyset\right\}$ and $c=\|D F\|_{\overline{U_{q}}}$.

Proof. Choose discrete subsets $D_{k} \subset Y \backslash V_{k}$ by

$$
D_{k}=E^{\prime}\left(\overline{U_{k-1}}, U_{k}, U_{k+1} ; \overline{V_{k-1}}, V_{k}, Y ; 1 / k, k+1+\#\left(\left(\cup_{i<k} D_{i}\right) \cap V_{k}\right)\right)
$$

and $D=\cup_{k} D_{k}$. Note that $D$ is discrete, because $D_{k} \subset Y \backslash V_{k}$.

Let $F: X \rightarrow Y$ be a holomorphic map which is non-degenerate, i.e. $\|D F\| \not \equiv 0$. Let $q \in \mathbb{N}$ be choosen in such a way that $F\left(\overline{U_{q}}\right) \cap \overline{V_{q}} \neq \emptyset$. Furthermore $c=\|\left. D F\right|_{\overline{U_{q}}}>0$. Finally assume that there is a number $M \in \mathbb{N}$ such that $\#\left(F\left(U_{n+1}\right) \cap D\right) \leq M+\#\left(V_{n} \cap D\right)$ holds for all $n \in \mathbb{N}$. Then for all $l>M$ we obtain

$$
\begin{aligned}
& \#\left(F\left(U_{l+1}\right) \cap D_{l}\right) \\
\leq & \#\left(F\left(U_{l+1}\right) \cap D\right) \\
\leq & M+1+\#\left(V_{l} \cap D\right) \\
< & l+1+\#\left(V_{l} \cap D\right)
\end{aligned}
$$

From the construction of $D$ it then follows that $F\left(U_{l}\right) \subset V_{l}$ for all $l>$ $\max (M, q+1,1 / c)$. Hence the statement of the proposition is fulfilled if we choose a map $\alpha$ in such a way that $\alpha(M, q, c) \geq \max \left(M, q+1, \frac{1}{c}\right)$ for all $(M, q, c) \in \mathbb{N} \times \mathbb{N} \times \mathbb{R}^{+}$.

\section{RIGID SETS}

For this section we fix the following presumptions.

Special Assumptions. 1. $(X, \rho)$ is weakly Stein (as defined in Def. 1).

2. $\left(r_{n}\right)_{n \in \mathbb{N}}$ is an strictly increasing sequence of positive numbers with $\lim r_{n}=+\infty$.

3. $U_{n}=\left\{x \in X: \rho(x)<r_{n}\right\}$.

4. $D$ is a discrete subset of $X$. 
5. $\alpha: \mathbb{N} \times \mathbb{N} \times \mathbb{R}^{+} \rightarrow \mathbb{N}$ is a map such that for every $f \in \operatorname{Aut}(X)$ the condition

$$
\# f\left(U_{n} \cap D\right) \leq m+\#\left(U_{n} \cap D\right) \forall n \in \mathbb{N}
$$

together with $f\left(\overline{U_{q}}\right) \cap \overline{U_{q}} \neq \emptyset$ implies that

$$
f\left(U_{n}\right) \subset f\left(U_{n+1}\right) \forall n \geq \alpha\left(m, q,\|D F\|_{\overline{U_{q}}}\right) .
$$

Prop. 2 above implies that for every weakly Stein manifold $(X, \rho)$ and every such sequence $\left(r_{n}\right)$ there exists a discrete subset $D$ and a map $\alpha$ such that these special assumptions are fulfilled.

Proposition 3. Under the special assumptions let $D^{\prime}$ be a subset of $X$ such that $\left(D \backslash D^{\prime}\right) \cup\left(D^{\prime} \backslash D\right)$ is finite.

Then $\operatorname{Aut}\left(X, D^{\prime}\right)=\left\{\phi \in \operatorname{Aut}(X): \phi\left(D^{\prime}\right)=D^{\prime}\right\}$ is a real Lie group (with countably many connected components).

Proof. The automorphism group $\operatorname{Aut}(X)$ embeds into $\operatorname{Hol}(X, X) \times$ $\operatorname{Hol}(X, X)$ via $\phi \mapsto\left(\phi, \phi^{-1}\right)$. We equip $\operatorname{Aut}(X)$ with the relative topology corresponding to this embedding $(\operatorname{Hol}(X, X)$ being topologized with the compact-open-topology). The topology on $\operatorname{Aut}\left(X, D^{\prime}\right)=$ $\left\{\phi \in \operatorname{Aut}(X): \phi\left(D^{\prime}\right)=D^{\prime}\right\}$ is induced by the inclusion $\operatorname{Aut}\left(X, D^{\prime}\right) \subset$ $\operatorname{Aut}(X)$.

Consider

$$
W=\left\{\phi \in \operatorname{Aut}\left(X, D^{\prime}\right): \phi\left(\overline{U_{1}}\right) \subset \overline{U_{2}} \text { and } \phi^{-1}\left(\overline{U_{1}}\right) \subset \overline{U_{2}}\right\}
$$

We will prove that $W$ is compact. For this note that

$$
\|D \phi\|_{p}=\left\|D \phi^{-1}\right\|_{\phi(p)}^{-1}
$$

for all $p \in X, \phi \in \operatorname{Aut}(X)$. Now $\phi\left(\overline{U_{2}}\right) \cap \overline{U_{2}} \neq \emptyset$ for $\phi \in W$. Therefore

$$
\|D \phi\|_{\bar{U}_{2}} \cdot\left\|D\left(\phi^{-1}\right)\right\|_{\bar{U}_{2}} \geq 1
$$

for all $\phi \in W$. Let $\left(\phi_{l}\right)_{l}$ be a sequence in $W$. By passing to a subsequence and possibly replacing $\left(\phi_{l}\right)_{l}$ by the sequence of the respective inverse maps $\left(\phi_{l}^{-1}\right)_{l}$, we may assume that $\left\|\left(D \phi_{l}\right)\right\|_{\bar{U}_{2}} \geq 1$ for all $l$. Furthermore observe that

$$
\begin{aligned}
& \# \phi_{l}\left(U_{n} \cap D\right) \\
\leq & \#\left(D \backslash D^{\prime}\right)+\# \phi_{l}\left(U_{n} \cap D^{\prime}\right) \\
= & \#\left(D \backslash D^{\prime}\right)+\#\left(U_{n} \cap D^{\prime}\right) \\
\leq & \#\left(D \backslash D^{\prime}\right)+\#\left(D^{\prime} \backslash D\right)+\#\left(U_{n} \cap D\right) .
\end{aligned}
$$

Hence $\phi_{l}\left(U_{n}\right) \subset U_{n+1}$ for all $l$ and $n$ with $n \geq N=\alpha\left(\#\left(D \backslash D^{\prime}\right)+\#\left(D^{\prime} \backslash\right.\right.$ $D), 2,1)$ by special assumption (5). Note that $N$ does not depend on $l$. Therefore a subsequence of $\left(\phi_{l}\right)_{l}$ converges to a holomorphic map $F: X \rightarrow X$. This convergence implies $\lim \sup _{l}\left\|D \phi_{l}\right\|_{\bar{U}_{2}}=\zeta<\infty$. 
Hence $\phi_{l}^{-1}\left(U_{n}\right) \subset U_{n+1}$ for $n \geq \alpha\left(\#\left(D \backslash D^{\prime}\right)+\#\left(D^{\prime} \backslash D\right), 2, \zeta\right)$ and we obtain that the sequence of inverse maps $\phi_{l}^{-1}$ converges as well. But now $\lim \phi_{l}=F$ and $\lim \phi_{l}^{-1}=G$ imply that $F \circ G=i d_{X}$. Hence $F, G \in \operatorname{Aut}(X)$. Furthermore it is clear that $F\left(D^{\prime}\right) \subset D^{\prime}$ and $G\left(D^{\prime}\right) \subset$ $D^{\prime}$. It follows that $F, G \in \operatorname{Aut}\left(X, D^{\prime}\right)$. Thus $W$ is compact. Since $W$ contains an open neighbourhood of $i d_{X}$ in $\operatorname{Aut}\left(X, D^{\prime}\right)$, it follows that $\operatorname{Aut}\left(X, D^{\prime}\right)$ is a locally compact topological group. By a result of Bochner and Montgomery [1] it follows that $\operatorname{Aut}\left(X, D^{\prime}\right)$ is a real Lie group.

Finally note that $X$ having countable base of topology implies that $\operatorname{Hol}(X, X)$ and consequently $\operatorname{Aut}\left(X, D^{\prime}\right)$ have likewise topologies with countable base. Since $\operatorname{Aut}\left(X, D^{\prime}\right)$ is a Lie group, its connected components are open. Hence the countability of the base of the topology implies that $\operatorname{Aut}\left(X, D^{\prime}\right)$ has only countably many connected components.

Remark. This evidently implies that the isotropy groups Aut $\left(X, D^{\prime}\right)_{p}$ $=\left\{g \in \operatorname{Aut}\left(X, D^{\prime}\right): g(p)=p\right\}$ are likewise Lie groups with countably many connected components. By arguments similar to the ones used in the above proof one may show in addition that $\operatorname{Aut}\left(X, D^{\prime}\right)_{p}$ is actually compact for all $p \in X$.

Lemma 5. Let $K$ be a real Lie group (with countably many connected components) acting effectively on a connected complex manifold by biholomorphic transformations.

Then there exists a finite set $S$ such that $k \in K, k(x)=x$ for all $x \in S$ implies $k=e$.

Proof. Let $n=\operatorname{dim} K$ and choose $x_{1}, \ldots x_{n}$ in such a way that

$$
\operatorname{dim}\left\{g \in K: g\left(x_{i}\right)=x_{i} \forall 1 \leq i \leq k\right\} \leq n-k .
$$

Then $\Gamma=\left\{g \in K: g\left(x_{i}\right)=x_{i} \forall 1 \leq i \leq n\right\}$ is discrete. For every $\gamma \in \Gamma$ the set $X^{\gamma}=\{x \in X: \gamma(x)=x\}$ is a closed analytic subset and therefore of measure zero. Thus $\cup_{\gamma \in \Gamma} X^{\gamma}$ is of measure zero, too. Now choose $S=\left\{x_{1}, \ldots, x_{n}, y\right\}$ with $y \notin \cup_{\gamma} X^{\gamma}$.

Proposition 4. Under the special assumptions there exists a finite set $S \subset X$ such that the following properties are fulfilled:

1. For every discrete subset $D^{\prime}$ of $X$ with $D \cup S \subset D^{\prime}$ the group Aut $\left(X, D^{\prime}\right)$ is countable.

2. If $\phi \in \operatorname{Aut}(X)$ and $\phi(x)=x$ for all $x \in D \cup S$, then $\phi=i d_{X}$.

Proof. Choose $p \in D$ and let $K=\operatorname{Aut}(X, D)_{p}$, i.e. $K=\{\phi \in \operatorname{Aut}(X)$ : $\phi(D)=D$ and $\phi(p)=p\}$. Then $K$ is a real Lie group acting effectively 
on the manifold $X \backslash D$. By the preceding lemma there is a finite set $S \subset X \backslash D$ such that $\left.k\right|_{S}=i d_{S}$ implies $k=i d_{X}$ for $k \in K$.

Now let $D^{\prime}$ be a discrete subset of $X$ with $D \cup S \subset D^{\prime}$ and let $G$ be the connected component of $i d_{X}$ in $\operatorname{Aut}\left(X, D^{\prime}\right)$. Since $G$ is connected and stabilizes $D^{\prime}$, it must act trivially on $D^{\prime}$. Hence $G \subset K$ and therefore $G=\{i d\}$ by the choice of $S$. It follows that $\operatorname{Aut}\left(X, D^{\prime}\right)$ is countable.

Finally, if $\phi \in \operatorname{Aut}(X)$ with $\left.\phi\right|_{D \cup S}=i d_{D \cup S}$, then $\phi \in K$ and thus $\phi=i d_{X}$ by the choice of $S$.

Theorem 2. Let $X$ be a weakly Stein manifold.

Then there exists a discrete subset $\Lambda$ with the property that $\phi \in$ $\operatorname{Aut}(X)$ with $\phi(\Lambda)=\Lambda$ implies $\phi=i d_{X}$.

Proof. Choose a finite set $S$ as in the above proposition. Recall that the choice of $S$ is generic, i.e. there is no problem in finding another finite subset $S^{\prime}$ with $S \cap S^{\prime}=\emptyset$ enjoying the same properties as $S$.

Let $D_{1}=D \cup S \cup S^{\prime}$. Then for every $x \in D$ we have the conclusion that $\operatorname{Aut}\left(X, D_{1} \backslash\{x\}\right)$ is countable and that $\left.\phi\right|_{D_{1} \backslash\{x\}}=i d$ implies $\phi=$ $i d_{X}$.

Let $\Omega$ be the set of all $\omega \in X \backslash D_{1}$ for which there exists an automorphism $\phi \in \operatorname{Aut}(X)$ such that $\phi$ stabilizes $D_{1} \cup\{\omega\}$ but $\phi(\omega) \neq \omega$. For every $\omega \in \Omega$ let us fix one such $\phi$, henceforth called $\phi_{\omega}$. For every $(a, b) \in D_{1} \times D_{1}$ let $\Omega(a, b)=\left\{\omega \in \Omega: \phi_{\omega}(\omega)=a\right.$ and $\left.\phi_{\omega}(b)=\omega\right\}$ and for every non-empty $\Omega(a, b)$ fix an element $\eta(a, b) \in \Omega(a, b)$. Then we obtain a map from $\Omega(a, b)$ to $A u t\left(X, D_{1} \backslash\{b\}\right)$ by

$$
\omega \mapsto \phi_{\omega}^{-1} \circ \phi_{\eta(a, b)}
$$

This map is injective, because

$$
\left.\left(\phi_{\omega}^{-1} \circ \phi_{\eta(a, b)}\right)\right|_{D_{1} \backslash\{b\}}=\left.\left(\phi_{\tilde{\omega}}^{-1} \circ \phi_{\eta(a, b)}\right)\right|_{D_{1} \backslash\{b\}}
$$

implies $\phi_{\omega}=\phi_{\tilde{\omega}}$ which in turn implies

$$
\omega=\phi_{\omega}(b)=\phi_{\tilde{\omega}}(b)=\tilde{\omega}
$$

It follows that $\Omega(a, b)$ is countable for all $(a, b) \in D_{1} \times D_{1}$. Therefore $\Omega$, being the union of all $\Omega(a, b)$, is countable as well.

For every $\phi \in A u t(X)$ the fixed point set $X^{\phi}$ is an analytic subset of $X$. Since $\operatorname{Aut}\left(X, D_{1}\right)$ is countable, it follows that

$$
\Omega^{\prime}=\left\{x \in X: \exists \phi \in \operatorname{Aut}\left(X, D_{1}\right) \backslash\{i d\}: \phi(x)=x\right\}
$$

is a set of measure zero.

Now $D_{1}, \Omega$ and $\Omega^{\prime}$ are sets of measure zero. Hence there is a point $p \in X$ contained in none of these three sets. Let $\Lambda=D_{1} \cup\{p\}$. We claim that $\operatorname{Aut}(X, \Lambda)=\{i d\}$. Indeed, $p \notin \Omega$ implies that there is no $\phi \in \operatorname{Aut}(X, \Lambda)$ with $\phi(p) \neq p$. Hence $\operatorname{Aut}(X, \Lambda) \subset \operatorname{Aut}\left(X, D_{1}\right)$. But 
$p \notin \Omega^{\prime}$ implies that $\phi(p) \neq p$ for all $\phi$ in $\operatorname{Aut}\left(X, D_{1}\right)$ except $i d_{X}$. It follows that $\operatorname{Aut}(X, \Lambda)=\{i d\}$.

The assumption of $X$ being weakly Stein was used to derive that certain subgroups of $A u t(X)$ are small in a certain sense. Therefore we may replace the assumption of $X$ being weakly Stein by the assumption that $\operatorname{Aut}(X)$ is sufficiently small, e.g. a Lie group. One can check that the arguments used in the proof of the theorem above also imply the result stated below.

Proposition 5. Let $X$ be a complex manifold, $\operatorname{dim}(X)>0$, such that Aut $(X)$ is a real Lie group.

Then there exists a discrete subset $D \subset X$ such that $\phi(D) \subset D$ implies $\phi=i d_{X}$ for $\phi \in A u t(X)$.

For certain complex manifolds $X$ it is true that $A u t(X)$ must be a real Lie group, e.g. this is true for compact complex manifolds as well as for bounded domains in $\mathbb{C}^{n}$ and other hyperbolic complex manifolds.

However, in general the automorphism group of a complex manifold does not need to be a Lie group. E.g., if $X$ is a connected complex manifold and $Y$ is a complex manifold with a complex one-parametergroup of automorphisms $\psi_{t} \in \operatorname{Aut}(Y)$ then for a every holomorphic function $f$ on $X$ a holomorphic automorphism of $X \times Y$ is given by

$$
(x, y) \mapsto\left(x, \psi_{f(x)}(y)\right) .
$$

This implies that $A u t(X, Y)$ can not be a Lie group unless every holomorphic function on $X$ is constant.

In this spirit we derive the following example.

Example 1. Let $Z$ be a compact complex manifold on which a complex Lie group $G$ acts effectively, $Y$ a Stein manifold and $X=Y \times Z$ (with $\operatorname{dim}(G), \operatorname{dim}(Y)>0)$. Then for every discrete subset $E \subset X$ there exists a non-trivial automorphism $\phi \in \operatorname{Aut}(X)$ with $\left.\phi\right|_{E}=i d_{E}$.

Proof. Let $\pi_{2}: X \rightarrow Y$ denote the projection onto the second factor. This map is proper, hence $\pi_{2}(E)$ is discrete in $Y$. Now simply choose a non-zero holomorphic function $f \in \mathcal{O}(Y)$ vanishing on $\pi_{2}(E)$ and an element $v \in \operatorname{Lie}(G)$ and let $\phi$ be the automorphism of $X$ defined by $(z, y) \mapsto(\exp (v f(y))(z), y)$.

\section{INEQUIVALENT DISCRETE SUBSETS}

The methods used in the preceding sections also may be employed to show the following. 
Theorem 3. Let $X$ be a weakly Stein manifold.

Then there exist uncountably many pairwise inequivalent infinite discrete subsets of $X$.

Here two discrete subsets $D, D^{\prime}$ of $X$ are called equivalent if there exists an automorphism $\phi \in \operatorname{Aut}(X)$ such that $\phi(D)=D^{\prime}$.

Proof. Let $D_{1}, \Omega$ and $\Omega^{\prime}$ be as in the proof of theorem 2. Let $\Sigma=$ $D_{1} \cup \Omega \cup \Omega^{\prime}$. For $x, y \in X \backslash \Sigma$ we define an equivalence relation by setting $x \sim y$ iff there exists an automorphism $\phi \in A u t(X)$ such that $\phi\left(D_{1} \cup\{x\}\right)=D_{1} \cup\{y\}$. For $x \in X \backslash \Sigma$ and $a \in D_{1}$ let

$E_{a}(x)=\left\{y \in X \backslash \Sigma: \exists \phi \in \operatorname{Aut}(X): \phi\left(D_{1} \cup\{x\}\right)=D_{1} \cup\{y\}, \phi(a)=y\right\}$

and

$$
E^{*}(x)=\left\{y \in X \backslash \Sigma: \exists \phi \in \operatorname{Aut}\left(X, D_{1}\right): \phi(x)=y\right\}
$$

Recall that $\operatorname{Aut}\left(X, D_{1}\right)$ is countable. Hence $E^{*}(x)$ is also countable. Let $y, \tilde{y} \in E_{a}(x)$ and let $\phi, \tilde{\phi}$ be corresponding elements in $\operatorname{Aut}(X)$. Then $\psi=\tilde{\phi} \circ\left(\phi^{-1}\right) \in \operatorname{Aut}(X)$ with $\psi(y)=\tilde{y}$ and $\psi\left(D_{1}\right)=D_{1}$. Thus countability of $\operatorname{Aut}\left(X, D_{1}\right)$ also implies that all the sets $E_{a}(x)$ are countable. Therefore the sets $E_{a}(x)$ and $E^{*}(x)$ are all countable as well. It follows that the equivalence classes of $\sim$ in $X \backslash \Sigma$ are countable. Since $X \backslash \Sigma$ is not countable, this implies that there are uncountably many equivalence classes.

In fact this result can be proved for many other spaces as well. For instance there is the following proposition.

Proposition 6. Let $X$ be a complex manifold. Assume that there exists a non-constant holomorphic function $f$ on $X$.

Then there exist two inequivalent infinite discrete subsets of $X$.

Proof. Let $r=\sup \{|f(x)|: x \in X\}$. If $r<\infty$, then choose a sequence $x_{n}$ in $X$ with $\lim \left|f\left(x_{n}\right)\right|=r$ and let $a$ be an accumulation point of $f\left(x_{n}\right)$. In this case $1 /(f-a)$ is an unbounded holomorphic function on $X$. Thus it is clear that for some sequences $x_{n}$ in $X$ there is a holomorphic function $f$ such that $\left|f\left(x_{n}\right)\right|$ is unbounded. If there are sequences on which every holomorphic function is bounded, we found two inequivalent infinite discrete subsets. Thus we may assume that there is no such sequence, i.e., we may assume that $X$ is holomorphically convex and we may consider the Remmert-reduction $\pi: X \rightarrow Z$ (where $\pi$ is connected, surjective and proper and $Z$ is Stein).

If $\pi$ is not injective, there is an infinite discrete subset $S \subset X$ such that $\left.\pi\right|_{S}$ is not injective and such an infinite discrete subsets is not equivalent to any infinite discrete subset $S^{\prime} \subset X$ for which $\left.\pi\right|_{S^{\prime}}$ is 
injective. As a consequence, we may assume that $\pi$ is injective, i.e., that $X \simeq Z$, i.e., that $X$ is Stein.

Now the assertion follows from the preceding proposition.

Conjecture. For every complex space $X$ with $\operatorname{dim}(X)>0$ there exists a uncountable family of inequivalent discrete subsets of the same cardinality.

For non-compact spaces this conjecture is motivated by the above considerations. For a compact complex space $X$, the automorphism group $\operatorname{Aut}_{\mathcal{O}}(X)$ is a finite-dimensional complex Lie group $G$. Then, for every natural number $n$ with $n \operatorname{dim}_{\mathbb{C}}(X)>\operatorname{dim}_{\mathbb{C}}(G)$ the induced action of $\operatorname{Aut}_{\mathcal{O}}(X)$ on the $n$-fold symmetric product $S^{n}(X)$ can not have an open orbit. It follows that for such a number $n$ there are uncountably many inequivalent subsets of $X$ with cardinality $n$. Hence the conjecture holds for compact complex spaces.

\section{UNAVOIDABLE SETS}

Let $X, Y$ be complex manifolds. A subset $S \subset Y$ is called unavoidable for a family $\mathcal{H}$ of holomorphic mappings from $X$ to $Y$ if $f(X) \cap S \neq \emptyset$ for every $f \in \mathcal{H}$. Given a pair of complex manifolds $(X, Y)$ of the same dimension let $\operatorname{Hol}^{*}(X, Y)$ denote the set of non-degenerate holomorphic maps. Clearly, $\mathrm{Hol}^{*}(X, Y)$-unavoidable discrete subsets do not exist for arbitrary pairs of complex manifolds $(X, Y)$. For instance, assume that $X$ is a bounded domain in a Stein manifold. Then there exists a non-degenerate holomorphic map from $X$ to the unit ball $B_{n}$ in $\mathbb{C}^{n}$. As a consequence there is a non-degenerate holomorphic map from $X$ to $Y$ with $F(X) \cap D=\emptyset$ for every complex manifold $Y$ and every closed analytic subset $D \subset Y$.

It is also necessary to put some conditions on the target manifold, even if it is non-compact.

Example 2. Let $X$ be a Stein manifold of dimension $n, p \in \mathbb{P}_{n}, Y=$ $\mathbb{P}_{n} \backslash\{p\}$ and $D \subset Y$ a discrete subset.

Then there exists a non-degenerate holomorphic map $F: X \rightarrow Y$ with $F(X) \cap D=\emptyset$.

Proof. Let $H \subset \mathbb{P}_{n}$ be a hyperplane which does not intersect $D \cup\{p\}$. After changing coordinates, we may assume that $p=[1: 0: \ldots: 0]$ and $H=\left\{\left[x_{0}: \ldots: x_{n}\right]: x_{0}=0\right\}$. Let $\phi \in \operatorname{Aut}\left(\mathbb{P}_{n}\right)$ defined by

$$
\phi:\left[x_{0}: \ldots: x_{n}\right] \mapsto\left[x_{0}: \frac{1}{2} x_{1}: \ldots: \frac{1}{2} x_{n}\right] .
$$

Now for any open neighbourhood $W$ of $p$ in $\mathbb{P}_{n}$ the complement $\mathbb{P}_{n} \backslash W$ is compact and $D \cap\left(\mathbb{P}_{n} \backslash W\right)$ is finite. Hence for any open neighbourhood 
$W$ of $p$ in $\mathbb{P}_{n}$ there exists a number $N(W)$ such that $\phi^{N(W)}(D) \subset W$. It follows that there exists a biholomorphic map $\zeta$ from $\mathbb{C}^{n}$ onto some open neighbourhood of $p$ in $\mathbb{P}_{n}$ such that $\zeta\left(\mathbb{B}_{n}\right)$ contains all of $D$. Next choose a non-degenerate holomorphic map $H: X \rightarrow \mathbb{C}^{n}$ and a nondegenerate holomorphic map $G: \mathbb{C}^{n} \rightarrow \mathbb{C}^{n}$ such that $G\left(\mathbb{C}^{n}\right)$ does not intersect the unit ball in $\mathbb{C}^{n}$ (such a map exists by the Fatou-Bieberbach construction). Then $F=\zeta \circ G \circ H: X \rightarrow \mathbb{P}_{n} \backslash\{p\}=Y$ has the desired property $F(X) \cap D=\emptyset$.

Theorem 4. Let $X$ be an irreducible, reduced affine variety and $(Y, \rho)$ be weakly Stein.

Then there exists a discrete subset $D \subset Y$ such that $F(X) \cap D \neq \emptyset$ for every non-degenerate holomorphic map $F: X \rightarrow Y$.

Proof. By the "Noether Normalization Lemma" there exists a finite ramified covering $\pi: X \rightarrow \mathbb{C}^{n}$. Let $V_{n}=\{y \in Y: \rho(y)<n\}$ and $U_{n}=\left\{x \in X:\|\pi(x)\|<e^{n^{2}}\right\}$. By prop. 2 there exists a discrete subset $D \subset Y$ such that for every non-degenerate holomorphic map $F: X \rightarrow Y$ with $F(X) \cap D=\emptyset$ there is a number $N$ such that $F\left(U_{n}\right) \subset V_{n}$ for $n \geq N$. We claim that there can not exist such a map $F$. Consider the plurisubharmonic function $F^{*} \rho$ on $X$ and define a plurisubharmonic function $\xi$ on $\mathbb{C}^{n}$ by

$$
\xi(v)=\sum_{x \in \pi^{-1}(v)} F^{*} \rho(x)
$$

(This formula is to be understood literally where $\pi$ is unramified, in the ramification locus multiplicities have to be taken into account.) Now $F\left(U_{n}\right) \subset V_{n}$ for $n \gg 0$ implies that

$$
\limsup _{\|v\| \rightarrow \infty} \frac{\xi(v)}{\log \|v\|}=0
$$

But $M(r)=\sup _{\|v\| \leq r} \xi(v)$ is an increasing convex function in $\log r$ (see e.g. [7], prop. 1.4.b). For a real convex function $t \mapsto M(t)$ we have

$$
M(t) \leq \frac{n-1}{n} M(0)+\frac{1}{n} M(n t)
$$

for all $t, n \in \mathbb{R}^{+}$. Thus $\lim _{n \rightarrow \infty} \frac{M(n t)}{n t}=0$ implies $M(t) \leq M(0)$ for all $t>0$. This forces $\xi$ to be constant. Let $\xi \equiv C$. Recall that $F^{*} \rho \geq 0$. It follows that $C \geq F^{*} \rho$. But plurisubharmonic functions bounded from above on affine varieties are constant. Hence $F^{*} \rho$ must be constant, which contradicts the assumption that $F$ is non-degenerate. 


\section{DiscRete Sets With MeAsure hyperbolic COMPLEMENTS}

Theorem 5. Let $(Y, \rho)$ be a weakly Stein complex manifold.

Then there exists a discrete subset $D \subset Y$ such that $Y \backslash D$ is measurehyperbolic, i.e., the Kobayashi-Eisenman pseudovolume on $Y \backslash D$ is a volume.

Proof. We will apply lemma 1 . For this purpose, let $n=\operatorname{dim} Y, U_{2}=$ $\mathbb{B}_{n}=\left\{v \in \mathbb{C}^{n}:\|v\|<1\right\}, U_{1}=\frac{1}{2} \mathbb{B}_{n}, C=\{(0, \ldots, 0)\} \in \mathbb{B}_{n}, c=1$ and $Y_{k}=\{y \in Y: \rho(y)<k\}$. Furthermore we endow $Y$ with an hermitian metric $h$ and denote the corresponding volume form by $\omega$. Let the manifold $U_{2}=\mathbb{B}_{n}$ be endowed with the euclidean metric.

For each $k \in \mathbb{N}$, we choose a discrete subset $E_{k} \subset Y \backslash Y_{k}$ by invoking lemma 4 with $E_{k}=E^{\prime}\left(C, U_{1}, U_{2} ; \overline{Y_{k-1}}, Y_{k}, Y ; 1,1\right)$ and we define

$$
D=\cup_{k \in \mathbb{N}} E_{k}
$$

Fix a point $p \in Y \backslash D$. Evidently $p \in \overline{Y_{k-1}}$ for some $k \in \mathbb{N}$. Let $f: \mathbb{B}_{n} \rightarrow Y \backslash D$ be a holomorphic map with $f(0)=p$. Then $E_{k} \subset D$ implies $\|D f\|_{0}<1$ or $f\left(\frac{1}{2} \mathbb{B}_{n}\right) \subset Y_{k}$. For the Kobayashi-Eisenman pseudovolume $\mu_{Y \backslash D}$ it follows that

$$
\mu_{Y \backslash D} \geq \min \left\{\omega, \frac{1}{2^{n}} \mu_{Y_{k}}\right\}
$$

where $\mu_{Y_{k}}$ denote the Kobayashi-Eisenman pseudovolume of the complex manifold $Y_{k}$. Since $Y_{k}$ is taut and therefore measure-hyperbolic, it follows that $\mu_{Y \backslash D}(p)>0$ for all $p \in Y$, i.e., $Y \backslash D$ is measurehyperbolic.

Remark. A holomorphic map from $\mathbb{C}^{n}$ to a $n$-dimensional measure hyperbolic complex manifold must be degenerate. Thus we reproved theorem $\$$ for the special case $X=\mathbb{C}^{n}$.

Example 3. Let $Y=\mathbb{P}_{2} \backslash\{[1: 0: 0]\}$. Then $Y \backslash D$ is never measure hyperbolic for any discrete subset $D \subset Y$. To verify this, note that there exists a non-degenarate holomorphic map from $\mathbb{C}^{n}$ to $Y \backslash D$ (see ex. Q) and recall that a holomorphic map from $\mathbb{C}^{n}$ to a measure hyperbolic manifold is necessarily degenerate.

\section{REFERENCES}

[1] Bochner, S.; Montgomery, D.: Locally Compact groups of differentiable Transformations. Ann. Math. 47 (4), 639-653 (1946)

[2] Brody, R.: Compact manifolds and hyperbolicity. T.A.M.S. 235 , 213-219 (1978)

[3] Eisenman, D.A.: Intrinsic measure on complex manifolds and holomorphic mappings. Mem. A.M.S. 96. Providence, R.I. 1970. 
[4] Kaliman, S.: Some facts about Eisenman intrinsic measures. Complex Variables Theory Appl. 27, 163-173 (1995)

[5] Kobayashi, S.: Intrinsic distances, measures and geometric function theory. Bull.AMS 82no.3, 357-416 (1976) MR 54 \# 3032

[6] Lang, S.: Introduction to Complex Hyperbolic Spaces. Springer 1987.

[7] Lelong, P.; Gruman, L.: Entire Functions of Several Complex Variables. Springer 1986.

[8] Rosay, J.P.; Rudin, W.: Holomorphic maps from $\mathbb{C}^{n}$ to $\mathbb{C}^{n}$. Trans. A.M.S. 310, 47-86 (1988)

[9] Wu, H.H.: Normal Families of Holomorphic Mappings. Acta Math. 119,194233 (1967)

Jörg Winkelmann, Universität Basel, Mathematisches Institut, RheinSPRUNG 21, CH-4051 BASEL, Switzerland

E-mail address: jwinkel@member.ams.org

Webpage: http://www.math.unibas.ch/ $\sim$ winkel/ 